\title{
Quantitative Convergence Between Physical-Chemical Constants of the Proton and the Properties of Water: Implications for Sequestered Magnetic Fields and a Universal Quantity
}

\author{
Michael A. Persinger \\ Laurentian University, 935 Ramsey Lake Road, Sudbury, Ontario P3E 2C6, Canada \\ E-mail address: mpersinger@laurentianl.ca
}

\begin{abstract}
The ratio of the magnetic moment and charge of a proton when multiplied by the viscosity of water results in forces that when applied over the distance of $\mathrm{O}-\mathrm{H}$ bonds provides quantum increments in the order of $10^{-20} \mathrm{~J}$. Precise coefficients of this order of magnitude are consistent with the mechanisms associated with proton $\left(\mathrm{H}^{+)}\right.$mobility and duration of the hydronium atom. When applied to aggregate properties of water that involve exclusion zones defined by boundaries containing marked proton density and coherent domains within which specific patterns of applied magnetic fields can be contained for protracted periods, these intrinsic properties suggest that the major features of the cell plasma membrane and living systems can be accommodated by proton movements within water. Water exposed in the dark to weak magnetic fields displayed a $\sim 10 \mathrm{~nm}$ shift in peak wavelength as measured by a fluorescence spectrophotometer. Given the persistent emergence of $10^{-20} \mathrm{~J}$ as a functional unit of energy across the universe, the physical significance of the interaction between weak, temporally patterned magnetic fields and the organization of water within astronomical and abiogenic contexts may have been underestimated.
\end{abstract}

Keywords: proton magnetic moment; hydronium ion; aggregate water properties; magnetic fields; spectrophotometry; $10^{-20} \mathrm{~J}$; cosmological parameters

\section{INTRODUCTION}

Discerning the quantitative bases for the physical-chemical properties of water is important to relate the different levels of scientific discourse. In the tradition of Bohr and others [1] the basic properties of the unit particles, the electron and proton, should be reflected in the spatial and temporal characteristics of larger aggregates. Establishing the quantitative relationships between the properties of water, the primary constituent of living systems and the most voluminous solvent on the planet, and the fundamental constants that define the characteristics of matter may facilitate our understanding of the mechanisms and processes by 
which mass and energy interact within this medium. Here I present quantitative evidence that the basic physical units predict the major properties of water that define living systems and that the energies associated with these interactions could reflect universal constants.

\section{PROTON PROPERTIES AND THE UNIVERSAL QUANTAL UNIT}

Dividing the magnetic moment of a proton $\left(1.41 \cdot 10^{-26} \mathrm{~A} \cdot \mathrm{m}^{2}\right)$ by the unit charge $\left(1.6 \cdot 10^{-}\right.$ ${ }^{19} \mathrm{~A} \cdot \mathrm{s}$ ) displayed by a proton (or an electron) results in a term of diffusion which is $0.88 \cdot 10^{-7}$ $\mathrm{m}^{2} \cdot \mathrm{s}^{-1}$. When applied to the average viscosity of water $\left(8.94 \cdot 10^{-4} \mathrm{~kg} \cdot \mathrm{m}^{-1} \mathrm{~s}^{-1}\right)$ around $25^{\circ} \mathrm{C}$ the force is $7.87 \cdot 10^{-11} \mathrm{~kg} \cdot \mathrm{m} \cdot \mathrm{s}^{-2}$.

If this force is applied over the distance of two $\mathrm{O}-\mathrm{H}$ bonds $\left(1.92 \cdot 10^{-10} \mathrm{~m}\right)$ that would constitute a water molecule the intrinsic energy would be $1.5 \cdot 10^{-20} \mathrm{~J}$. For comparison, the resulting quantity of energy for biological temperatures $\left(37^{\circ} \mathrm{C}\right)$ where viscosity is $\sim 6.3 \cdot 10^{-3}$ $\mathrm{Pa} \cdot \mathrm{s}$, is $1.1 \cdot 10^{-20} \mathrm{~J}$ and at $20^{\circ} \mathrm{C}$ (the classic standard temperature) where viscosity is $1.00 \cdot 10^{-3}$ $\mathrm{Pa} \cdot \mathrm{s}$ the energy would be $1.7 \cdot 10^{-20} \mathrm{~J}$.

The order of magnitude of $10^{-20} \mathrm{~J}$ is relevant for several reasons. First it is the quantum of energy associated with the resting membrane potential produced by the electrical force between potassium ions each separated by about $11 \mathrm{~nm}$ over the surface of the membrane [2]. Second, this magnitude defines the energy associated with the effects of the net change in voltage of an action potential $\left(\sim 1.2 \cdot 10^{-1} \mathrm{~V}\right)$ on a unit charge.

Third, the energy is within measurement error for the second shell hydrogen bonds $(2.6$ $\mathrm{kcal} \cdot \mathrm{M}^{-1}$ ) that have been shown to be primarily contributory to the capacity for proton mobility [3] within water.

As aptly articulated by Decoursey [3] the proton is unique among cations because it is interchangeable with the protons that form water. Consequently water maintains its structure while the constituent protons move through space and time. This capacity is coupled to a very small concentration of free protons $\left(\mathrm{H}_{3} \mathrm{O}^{+}\right)$, the hydronium ion, particularly in physiological systems. Whereas the concentration of $\mathrm{H}^{+}$in water is $110 \mathrm{M}$, the concentration of hydronium ions is $\sim 40 \mathrm{nM}$. Only one proton in about a billion comprises $\mathrm{H}_{3} \mathrm{O}^{+}$at any given instant [3] .

The average life time of the $\mathrm{H}_{3} \mathrm{O}^{+}$ion has been estimated by more than a dozen researchers to range between 0.24 to 3 ps with a median of $\sim 1$ ps. When this temporal parameter $\left(10^{-12} \mathrm{~s}\right)$ is multiplied by the ratio of the proton's magnetic moment and charge $\left(0.88 \cdot 10^{-7} \mathrm{~m}^{2} \cdot \mathrm{s}^{-1}\right)$, the resulting area is $8.8 \cdot 10^{-20} \mathrm{~m}^{2}$, or $2.97 \cdot 10^{-10} \mathrm{~m}(0.297 \mathrm{~nm})$.

The actual distance between water molecules is usually measured as 2.9 A or $0.29 \mathrm{~nm}$. In other words, the duration of the hydronium ion is coupled to the diffusivity of the dynamics of $\mathrm{H}_{2} \mathrm{O}$.

Proton mobility in water, which is $\sim 3.6 \cdot 10^{-7} \mathrm{~m}^{2} \cdot \mathrm{V}^{-1} \cdot \mathrm{s}^{-1}$ [3], has been shown quantitatively to be related to the movement of photons through tissue, including the human brain [4] through a Grotthuss chain-like effect associated with free protons.

The subtle effects of weak, extremely low frequency magnetic fields generated from the geophysical environment within the cerebral tissue upon water containing physiological concentrations of ions may be primarily mediated by these proton movements [5].

Recently we have shown that experimentally-induced shifts in $\mathrm{pH}$ between two nonlocal reactions that shared weak magnetic fields $(\sim 1 \mu \mathrm{T})$ with specific temporal parameters for changing, accelerating and decelerating angular velocities exhibited the conspicuous excess correlation that has defined entanglement [6]. 


\section{RELEVANCE TO AGgREgRATE PROPERTIES}

Water molecules do not behave only as singular entities whose primary structure remains remarkably stable. Large numbers of water molecules exist for short periods as clusters whose numbers and structures depend upon ambient temperature. Flickering clusters with durations of $\sim 10^{-11} \mathrm{~s}$ have been estimated to contain $\sim 50$ molecules at $20{ }^{\circ} \mathrm{C}$ and 40 molecules at $37^{\circ} \mathrm{C}$. Above $35^{\circ} \mathrm{C}$ the clusters display configurations more typical of networks [7].

The spatial order of water adjacent to hydrophilic surface areas, interfacial water, differs remarkably from bulk water. The existence of "exclusion zones" whereby colloidal and molecular solutes suspended in aqueous solutions are profoundly excluded from the vicinity of the surface has been shown by Pollack and his colleagues [8]. These widths of solute free zones were at least $100 \mu \mathrm{m}$ in width. Within the exclusion zones there was a 10 fold increase in viscosity. Between the exclusion zone and the bulk water the potential difference was within the order of $100 \mathrm{mV}$ (up to $150 \mathrm{mV}$ ). This EZ-bulk water boundary was occupied by protons. The most conspicuous implication of these measurements was that the properties of the exclusion zones of interfacial water might be the primary bases of living systems that has been traditionally attributed exclusively to the plasma cell membrane. This would suggest that the primary role of the physical lipoprotein boundary that defines the cell membrane would be to ensure spatial-temporal stability of these functions rather than causing their occurrences.

Chai et al al [9] examined the absorption and fluorescence characteristics of aqueous solutions of sugars, salts, and amino acids by employing UV spectroscopy and spectrofluorometry. They found clear evidence of peak emissions of photons with wavelengths $\sim 270 \mathrm{~nm}$ within the exclusion zones adjacent to various hydrophilic surfaces. This empirical observation is congruent with the intrinsic force associated with the product of viscosity and the diffusivity ratio for the magnetic moment of the proton and charge developed in the present paper.

For example, when the force $\left(7.87 \cdot 10^{-11} \mathrm{~N}\right)$ associated with the product of the viscosity of water and the ratio of the magnetic moment to unit charge of the proton is applied across a plasma membrane (assuming $10 \mathrm{~nm}$ ) the energy would be $7.87 \cdot 10^{-19} \mathrm{~J}$ or a frequency equivalence, when divided by Planck's constant $\left(6.626 \cdot 10^{-34} \mathrm{~J} \cdot \mathrm{s}^{-1}\right)$ of $1.19 \cdot 10^{15} \mathrm{~Hz}$. Assuming $\mathrm{c}$, the velocity of light in a vacuum, the peak wavelength would be $\sim 252 \mathrm{~nm}$. This is within measurement error and statistical dispersions for the ranges of experimental temperatures that were reported by Chai et al [9] to be emitted from exclusion zones along hydrophilic surfaces. More precisely, if the functional width of the membrane were $9.3 \mathrm{~nm}$ the peak wavelength would be $\sim 270 \mathrm{~nm}$, which was their empirical result. This solution, if valid, would couple quantum phenomena (by Planck's relations) to the fundamental units (the proton magnetic moment and charge) to the wavelength of electromagnetic energy (light) emitted by water molecules within this particular organization.

\section{INTERACTIONS WITH APPLIED MAGNETIC FIELDS}

Although the hydrogen bond comprises only $~ 5 \%$ of the $\mathrm{O}-\mathrm{H}$ bond energy it significantly determines the interactions between water molecules and their solutes [10]. With a typical range of 4 to $4.5 \mathrm{kCal}\left(16.74\right.$ to $\left.18.82 \cdot 10^{3} \mathrm{~J}\right)$ per mole or the equivalent electrostatic energy of between $2.75 \cdot 10^{-20} \mathrm{~J}$ to $3.1 \cdot 10^{-20} \mathrm{~J}$ per molecule, this means that the intrinsic energy is a factor of 2 greater than the energy applied from intrinsic forces over the distance of two 
O-H bonds. Although the precision of this doubling must be established, what is important is that only about 20 hydrogen-bonded sequences of water molecules would be required to extend the distance of the phospholipids that comprise the typical plasma membrane. Such a "water wire" has the capacity to conduct protons [3].

The dielectric relaxation time, the temporal latency between the onset of of an electric field and the induced polarization of water, displays a common activation energy of $4.6 \mathrm{Kcal}$ per mole. When the two $\mathrm{H}^{+}$that organize the polarity of water are accommodated this is equivalent to $1.6 \cdot 10^{-20} \mathrm{~J}$ as a quantum per hydrogen unit. Because voltage is energy divided by charge $\left(1.6 \cdot 10^{-20} \mathrm{~J}\right.$ divided by $\left.1.6 \cdot 10^{-19} \mathrm{~A} \cdot \mathrm{s}\right)$, the resulting intrinsic value would be $100 \mathrm{mV}$. This quantity is the median value for the potential difference generated between bulk water and interfacial water that has been attributed to the shell of protons that separate the boundaries.

The rotational relaxation time ("time constant, $\tau$ ") of water can be estimated by:

$$
\tau=4 \pi \delta \mathrm{a}^{3}(\mathrm{kT})^{-1}
$$

where $\mathrm{a}=9.6 \cdot 10^{-11} \mathrm{~m}(0.96 \mathrm{~A})$ for the length of a single $\mathrm{O}-\mathrm{H}$ bond, which is the radius of that dipole, $\delta$ is the viscosity of water, $\mathrm{k}=$ the Boltzmann constant and $\mathrm{T}$ is temperature.

The quantification is:

$$
\left[(12.58) \cdot\left(8.94 \cdot 10^{-4} \mathrm{~Pa} \cdot \mathrm{s}\right) \cdot\left(9.6 \cdot 10^{-11} \mathrm{~m}\right)^{3}\right] \cdot\left[\left(1.38 \cdot 10^{-23} \mathrm{~J} \cdot \mathrm{T}^{-1}\right) \cdot\left(2.98 \cdot 10^{2} \mathrm{~K}\right)\right]^{-1} \text { or } 2.4 \cdot 10^{-12} \mathrm{~s} \text {. }
$$

This is within the range of the life time of the hydronium ion. For comparison the estimated time between "jumps" of a water molecule into a new position is estimated to be $\sim 4 \cdot 10^{-12} \mathrm{~s}$. Stated alternatively, the relaxation time of water (when an electric field associated with the application of an external magnetic field is considered) results in the duration of the life time of a hydronium ion. This strongly suggests that application of magnetic energy within a volume of water could be mediated through the ubiquitous and continuous movements of protons through the water matrix. Integrating the rationale concerning the nature of the cell membrane from the previous section, one would predict that the appropriately patterned magnetic field could displace energetic wavelengths within the range of the visible spectrum by the width of a membrane.

We have recently demonstrated this effect. Murugan et al [11] have demonstrated a reliable and robust shift of $\sim 10 \mathrm{~nm}$ in the peak fluorescent wavelength of light through $1 \mathrm{cc}$ aliquots of spring water that had been exposed, in $50 \mathrm{cc}$ containers within the dark, for 18 days to weak $(1 \mu \mathrm{T})$ magnetic fields with temporal structures that exhibit physiological patterns and intrinsic but complex frequency modulation. This is the same field that, in association with a second pattern, completed dissolved the aqueous flat worm: the planarian [12]. In multiple experiments sets of three beakers were placed at the edge of an active coil, at the edge of an inactive coil at a distance of $1 \mathrm{~m}$, and in a central position between the two. The three specific intensity ranges were high $(4.4$ to $11.5 \mu \mathrm{T})$, low $(0.11$ to $0.15 \mu \mathrm{T})$, and medium $(0.3$ to $0.6 \mu \mathrm{T})$, respectively. In each experiment a fourth beaker (control) was placed outside this geometry in the background intensity from ambient $60 \mathrm{~Hz}$ (power frequencies) of $0.1 \mu \mathrm{T}$.

We found that the critical temporal factor that determined the effects of this magnetic field upon photon emissions was the duration of the point durations that determined the sequential voltages generating the magnetic field pattern. The effect was only evident with 3 $\mathrm{ms}$ point durations but not with either 1 or $2 \mathrm{~ms}$ or 5 and $10 \mathrm{~ms}$ point durations. We have 
shown previously that $3 \mathrm{~ms}$ point durations are very likely to be associated with the intrinsic cosmological properties of the proton as inferred from Hubble's parameter [13].

Employing fluorescence spectrophotometry (Ultrospec 2100 pro uv visible spectrophotometer) with a stimulation wavelength of $250 \mathrm{~nm}$ we measured photon transmission over successive $1 \mathrm{~nm}$ wavelengths between 250 and $500 \mathrm{~nm}$. An example of these results is shown in Figure 1. It shows the mean values of photon counts for triplicate replications for each successive $1 \mathrm{~nm} \lambda$ between 350 and $466 \mathrm{~nm}$. The $10 \mathrm{~nm}$ shift to the longer wavelength occurred only in the water exposed to the $<0.3$ to $0.6 \mu \mathrm{T}$ fields but not to the more intense strengths. At this wavelength the shift of $10 \mathrm{~nm}$ is equivalent to a net change of $10^{-20} \mathrm{~J}$.

One interpretation is that continuous application of this magnetic field pattern to spring water in darkness altered the intrinsic organization of the protons. This condition particularly affected the distribution of energy from the second shell hydrogen bonds that contribute to proton movements. If classic processes are assumed, then a shift occurred such that different vibrational phases within the ground state were produced by the maintained exposure to the magnetic field pattern.

That the quantitative shift in the wavelength (and intrinsic energy) reflects magnetic energy contained within the spring water from the magnetic field exposures can be estimated by quantification. The net increase in photon counts $\mathrm{s}^{-1}$ for the medium intensity field was $\sim 100$ (Figure 1).

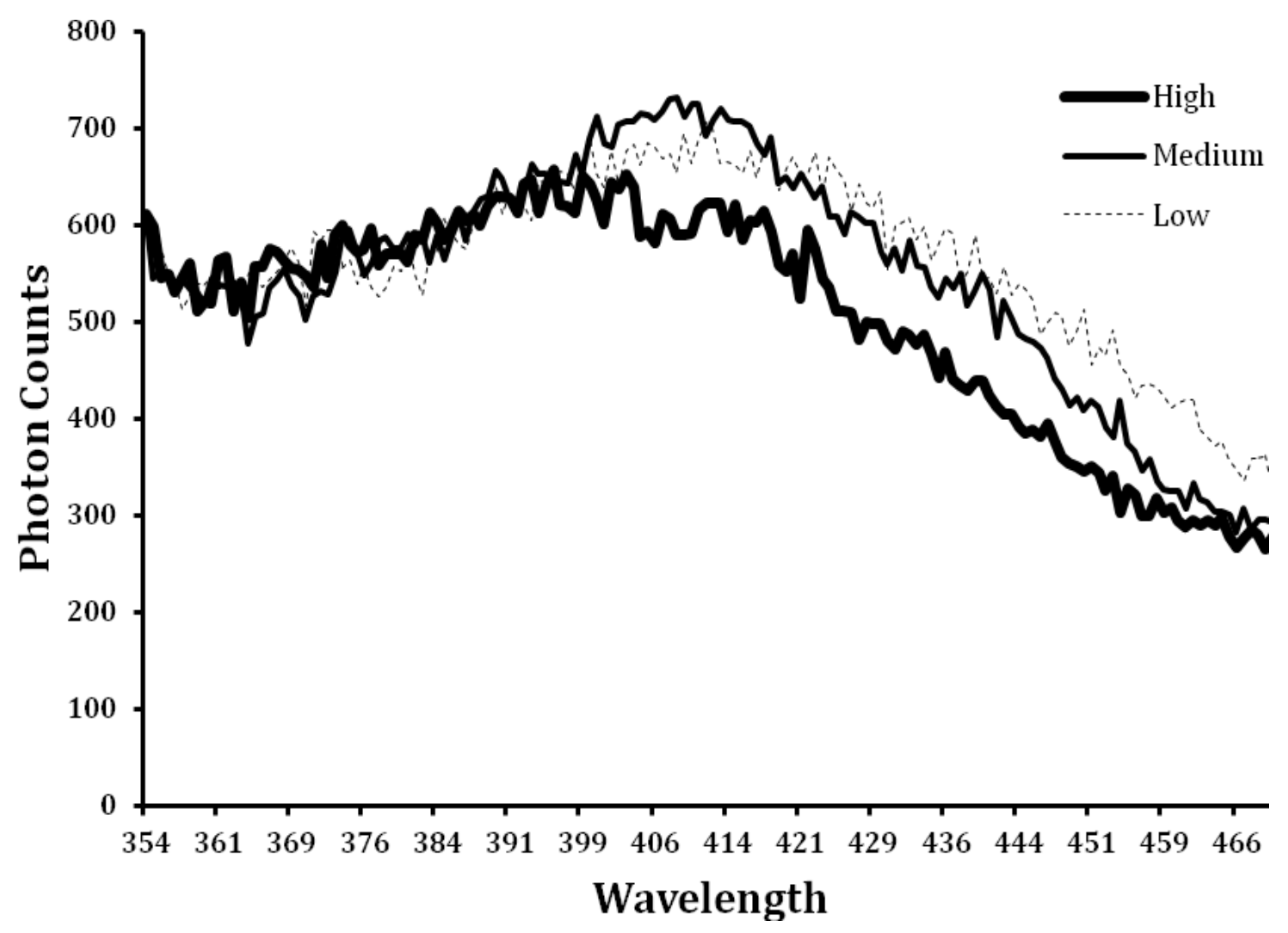

Figure 1. Photon counts from the fluorescence spectrophotometer over wavelengths through water that had been exposed to the high, medium, and low intensity magnetic fields in darkness. 
Assuming $5 \cdot 10^{-19} \mathrm{~J}$ per photon for the $\sim 400 \mathrm{~nm}$ wavelength range where the effect appears and the maintenance of this discrepancy over about 50,1 nm increments shifted to the right (Figure 1), the total energy emitted as photons would be $\sim 2.5 \cdot 10^{-15} \mathrm{~J}$. According to traditional estimates the magnetic energy within a medium is:

$$
\mathrm{J}=\mathrm{B}^{2} \cdot\left(2 \cdot 4 \pi \cdot 10^{-7} \mathrm{~N} \cdot \mathrm{A}^{-2}\right)^{-1} \cdot \mathrm{m}^{3}
$$

where the total energy from the medium strength field $\left(6 \cdot 10^{-8} \mathrm{~T}\right)$ in a $1 \mathrm{cc}\left(10^{-6} \mathrm{~m}^{3}\right)$ volume of water would be $1.4 \cdot 10^{-15} \mathrm{~J}$. This is well within the range of measurement error. Although clearly other experiments would be required to establish causality the convergence of the quantitative values for energy suggests the photon emissions reflected the Hamiltonian, the total energy within the system, from the exposure to the magnetic field.

The $10 \mathrm{~nm}$ shift is consistent with the more quantum-like phenomenon described by Del Giudice and Preparata [14]. They pursued the concept that above a given atomic density and below a specific temperature the Dicke Hamiltonian relation exhibited a spontaneous phase transition. Within this Superradiant Phase Transition (SPT) large classical electromagnetic fields become contained within arrangements of atomic "networks" and oscillate in phase with the atomic transitions between these specific states and the ground state. According to Del Giudice and Preparata, the energy gap between $\mathrm{N}$, two-level atoms is described as $\mathrm{E}=$ $h \cdot c \cdot \lambda^{-1}$, where $h$ is Planck's constant, $\mathrm{c}$ is the velocity of light and $\lambda$ is wavelength. Within the "coherent domain" whose width is $\lambda$, the two atomic systems vacillate between the two states in phase with the oscillation of the coherent electromagnetic field.

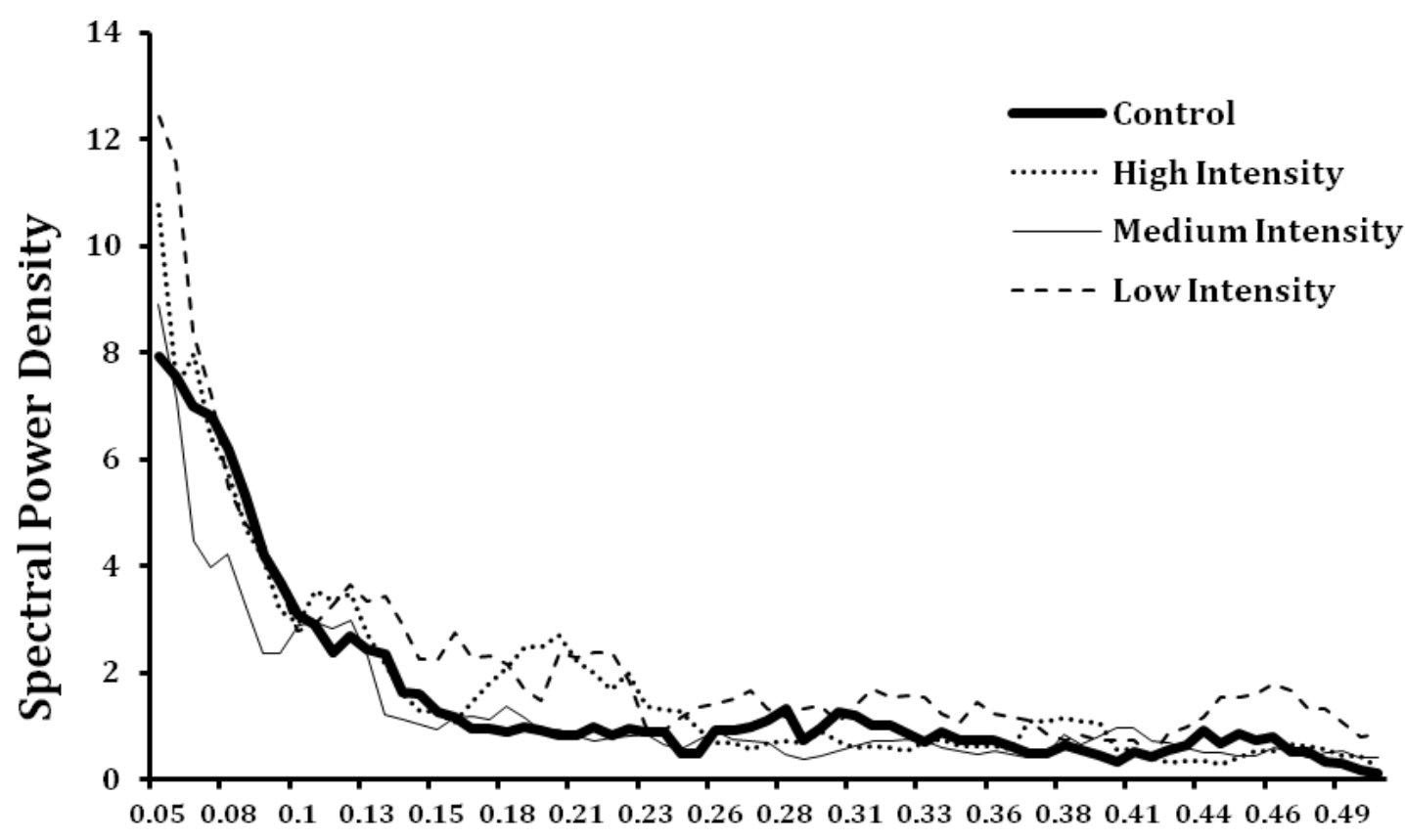

\section{Spectral Frequency}

Figure 2. Mean (of triplicates) of spectral power density for the successive $1 \mathrm{~nm}$ increments of photon emissions (Figure 1) for spring water that had been exposed in darkness to control, high intensity, medium intensity and low intensity, temporally pattermed magnetic fields. Note the symmetrical peak (small dotted line) around 0.2 spectral frequency $(5 \mathrm{~nm})$ for the high intensity exposures. 
When applied to our experiments the difference in energy, the gap, between $\lambda=400 \mathrm{~nm}$ and $\lambda=410 \mathrm{~nm}$ (peaks) is the difference between the two values $4.97 \cdot 10^{-19} \mathrm{~J}$ and $4.85 \cdot 10^{-19} \mathrm{~J}$, or, $1.2 \cdot 10^{-20} \mathrm{~J}$. If the electromagnetic field is "trapped" within the coherent domain one would expect more photon transmission and greater scatter to shift to the lower wavelengths as photons intrinsic to the water aggregates shift between states. This interpretation is consistent with the observations.

If an effect is coupled to fundamental physical processes, then coupled intrinsic patterns should emerge. Spectral analyses of the variations in photon emissions through the water that had been exposed to the magnetic fields revealed spectral power densities that peaked at spectral frequencies $\sim 0.1$ and 0.2. These results are shown in Figure 2. The actual wavelength is obtained by dividing 1 by the spectral frequency; these values are $10 \mathrm{~nm}$ and $5 \mathrm{~nm}$, respectively. The results indicate that there is a relative peak in spectral power density of the photon transmission through the water exposed to these magnetic fields that occurs every approximately $10 \mathrm{~nm}$ and $5 \mathrm{~nm}$.

The latter value approximates Del Guidice and Preparata's [14] derivation that the radius $\mathrm{R}$ of the coherent domain within which an "evanescent electromagnetic wave pulsates with the frequency $\omega^{\prime \prime}$, is $=3 / 8 \cdot \lambda$, effectively one-half a wavelength. In Figure 2 , water that was exposed to the higher intensity ( 4 to $12 \mu \mathrm{T}$ indicated by small dotted line) displays a clear rise, peak and fall in spectral density around $5 \mathrm{~nm}$.

\section{RELATIONSHIPS TO ASTROPHYSICAL AND COSMOLOGICAL PARAMTERS}

If the properties of water are derived from the basic properties of protons then these essential features of matter should reflect the characteristics contained within the entire universe. To paraphrase Ernst Mach, any change within local matter should be influenced by or could possibly reflect the entire universe. There are quantitative estimates to support this supposition.

When the total set is considered, there is no temporal term because processes or timerelated phenomena require at least two successive increments of time to be discerned (the Nyquist Limit). If the mass of the universe is assumed to be $\sim 10^{52} \mathrm{~kg}$ [15], the distance is $10^{26}$ $\mathrm{m}$ and the intrinsic frequency or oscillation of space (the Zwittterbewung) is $10^{86} \mathrm{~Hz}^{2}$, then the force would be $\sim 10^{164} \mathrm{~N}$. The smallest space to which it would be applied, Planck's length, has a cubic volume of $\sim 10^{-105} \mathrm{~m}^{3}$. This means that within a universe with a volume of $10^{78} \mathrm{~m}^{3}$ there would be $10^{183}$ equivalents of Planck's length "voxels". As a result the force would be $\left(10^{164} \mathrm{~N}\right.$ divided by $10^{183}$ voxels $) \sim 10^{-19} \mathrm{~N}$, per fundamental length of space. When this force is extended over the most frequent length constant of the universe, the hydrogen line of $21 \mathrm{~cm}$, the average unit energy is in the order of $\sim 10^{-20} \mathrm{~J}$.

This solution converges with the gravitational potential energy from the gravitational force between two photons, each with the upper limit rest mass of $10^{-52} \mathrm{~kg}$ [16]. The force between these two masses $\left(10^{-104} \mathrm{~kg}^{2}\right)$ if separated by the square of Planck's length $\left(10^{-70} \mathrm{~m}^{2}\right)$ and multiplied by $\mathrm{G}\left(6.6 \cdot 10^{-11} \mathrm{~m}^{3} \cdot \mathrm{kg}^{-1} \cdot \mathrm{s}^{-2}\right)$ would be $10^{-45} \mathrm{~N}$. When distributed over distance, for energy, that defines the radius of the visible universe, the energy is between $10^{-19}$ and $\sim 10^{-}$

${ }^{20} \mathrm{~J}$ depending if $8 \pi$ (the second derivative of the surface area of a sphere) is multiplied by $\mathrm{r}^{2}$ to accommodate basic curvature [17].

This is a relevant value for several reasons. First, the quantum is within the same order of magnitude as the essential bond strengths within water associated with proton movements and with the hydrogen bond which determines the majority of aqueous properties. Secondly, 
the gravitational force between two potassium ions separated by the distance $(11 \mathrm{~nm})$ reflecting the density of the layer of these ions that defines the resting membrane potential, is $\left(6.2 \cdot 10^{-26} \mathrm{~kg}\right)^{2}$ divided by $\left(1.1 \cdot 10^{-8} \mathrm{~m}\right)^{2}$ multiplied by $\mathrm{G}\left(6.67 \cdot 10^{-11} \mathrm{~m}^{3} \mathrm{~kg}^{-1} \mathrm{~s}^{-2}\right)$, or, $4.1 \cdot 10^{-46}$ $\mathrm{N}$. When multiplied over the distance of $10^{26} \mathrm{~m}$, the approximate width of the universe, the energy would be $\sim 10^{-20} \mathrm{~J}$. This would suggest that the energy from the upper limit of rest mass photons spread over the length of the universe would be equivalent in magnitude to the gravitational energy when the forces between $\mathrm{K}^{+}$ions that comprise the resting membrane potential are distributed over a comparable distance.

The specific shift in photon energies by $\sim 10 \mathrm{~nm}$ and the emergence of spectral perturbations within the range for the water that had been exposed to the magnetic fields in the dark may have astrophysical relevance. In some dense molecular clouds, such as the Orion Nebula, water is one of the most abundant molecules following molecular hydrogen and carbon monoxide [18]. The water density is $\sim 10^{7}$ molecules per $\mathrm{m}^{3}$ with a typical abundance ratio of $\left[\mathrm{H}_{2} \mathrm{O}\right] /\left[\mathrm{H}_{2}\right] \sim 10^{-5}$. According to Neronov and Vovk [19] the strength of magnetic fields in galaxies and galactic clusters is in the order of $10^{-10} \mathrm{~T}$. According to equation (2) the energy from this intensity field within $1 \mathrm{~m}^{3}$ of those molecules would be $5 \cdot 10^{-}$ ${ }^{15} \mathrm{~J}$, which is effectively the same value associated with the photons emitted from the water that had been exposed in the dark to the experimental magnetic fields. Given the demonstration of non-local, excess correlations ("entanglement") at substantial distances at the macrolevel for concentrations of $\mathrm{H}^{+}$in water [6] and between two single atoms [20], the issue of relative density may not be a critical factor that determines the interaction between interstellar water molecules.

\section{APPLICATION TO HUMAN CEREBRAL FUNCTION}

If the unit force associated with the product of the viscosity of water and the ratio of the proton's magnetic moment-to-charge is applied across the length of an average cerebrum (11 $\mathrm{cm}$ ), then the intrinsic energy for that volume of mass is $8.66 \cdot 10^{-12} \mathrm{~J}$. Within the volume of the human brain, of about $1.33 \cdot 10^{-3} \mathrm{~m}^{3}$, the "energy density" would be an average $6.5 \cdot 10^{-9} \mathrm{~J} \cdot \mathrm{m}^{-3}$. If each action potential is associated with a unit of energy of $\sim 2 \cdot 10^{-20} \mathrm{~J}$, then the numbers of these information-carrying events per implicit second could involve be $\sim 3 \cdot 10^{11}$ neurons. If each neuron was discharging at the known peak power of cerebral output of $\sim 10 \mathrm{~Hz}$, then the electromagnetic energy from these transient ion flows of 30 billion neurons would be accommodated. This estimate is within the order of magnitude of the numbers of neurons in the human cerebral cortices [21].

From a universal perspective this energy density is also convergent with the averaged energy within the volume of the known universe. Assuming the total energy of the universe as $\sim 10^{69} \mathrm{~J}$, which can be derived from a number of different approaches and assumptions [22], and the volume (based upon the current age) of $\sim 10^{78} \mathrm{~m}^{3}$, the average value within the whole set would be $10^{-9} \mathrm{~J} \cdot \mathrm{m}^{-3}$. Clearly the energy is not distributed homogeneously within spacetime. However if the average does reflect the units [23], similar to the observation that the plasma membrane's time-constant $(\sim 100 \mathrm{~ms}$, or $10 \mathrm{~Hz})$ of a typical neuronal system reflects the average temporal variation of the entire set (the cerebral cortices), then this convergence between available power density from the intrinsic energy from the physical-chemical bases of water applied across the length of the human cerebrum and the average density of all energy within the universe, including dark matter and energy, may have potential meaning. 
Cerebral function, particularly within the cerebral cortices, is related to recursive productions of rostral-caudal waves of electromagnetic fields integrated over remarkably large cortical areas that are recreated every 20 to $25 \mathrm{~ms}$. This " $40 \mathrm{~Hz}$ " field is considered to be the primary correlate of consciousness [24]. However there is also a phase velocity associated with this field that creates the additional property that could define some of the more intricate features of consciousness such as higher order awareness. Phase velocity is derived from:

$$
\sqrt{ }(4 \pi \mathrm{f}) \cdot(\mu \sigma)^{-1}
$$

where $\mathrm{f}=$ frequency, $\mu=$ magnetic permeability $\left(4 \pi \cdot 10^{-7} \mathrm{~N} \cdot \mathrm{A}^{-2}\right)$, and $\sigma=$ conductivity $\left(4 \mathrm{~S} \cdot \mathrm{m}^{-}\right.$ $\left.{ }^{1}\right)$. For $40 \mathrm{~Hz}$, the phase velocity is $10^{4} \mathrm{~m} \cdot \mathrm{s}^{-1}$. The time required for a quantity of energy to traverse a $10 \mathrm{~nm}$ length, such as a plasma membrane, at this velocity would be $\sim 10^{-12} \mathrm{~s}$, the median duration of a given positronium ion.

Finally there is a conspicuous physical cosmology to this connection. The average pressure within the universe assuming a density of 1 proton per $\mathrm{m}^{3}$ and the velocity of light in a vacuum is $1.44 \cdot 10^{-10} \mathrm{~Pa}$ [15]. The implicit velocity within water, assuming a density of $10^{3}$ $\mathrm{kg} \cdot \mathrm{m}^{-3}$ is $1.44 \cdot 10^{-13} \mathrm{~m}^{2} \cdot \mathrm{s}^{-2}$ or $3.79 \cdot 10^{-7} \mathrm{~m} \cdot \mathrm{s}^{-1}$. The time required for this process to traverse a plasma membrane of $10 \mathrm{~nm}$ is $\sim 26 \mathrm{msec}$. This is the average time involved with the recursive cerebral waves associated with consciousness.

\section{CONCLUSIONS}

The solutions for rational comparisons of quantitative values for universal constants of the proton within the context of the water and the presence of additional $\mathrm{H}^{+}$within this remarkable structure suggest that all of the major and apparently "complex" properties relevant to livings systems can be predicted from basic physical constants. The induction of exclusion zones whose properties differ from bulk water near surface boundaries and the emergence of coherent domains within radii within the $100 \mu \mathrm{m}$ range that can "trap" applied magnetic fields that in turn alter the intrinsic photon emissions, create the condition for the operation of "consciousness" and other complex functions. Because the fundamental quantum of energy associated with the physical-chemical dynamics of protons in water are within the same order of magnitude $\left(\sim 10^{-20} \mathrm{~J}\right)$ as the unit energy that could integrate energies distributed within all levels of space as well as gravitational-electromagnetic interactions, water on earth and within living systems, including the human brain, may reflect many of the intrinsic conditions within the universe at a local level.

\section{References}

[1] Sir W. Ramsay, F. G. Donnan, Text-Books of Physical Chemistry: A System of Physical Chemistry. Longmans, Green and Co. 1921.

[2] M. A. Persinger, Current Medicinal Chemistry 17 (2010) 3094-3098.

[3] T. E. Decoursey, Physiology Reviews 83 (2003) 475-579.

[4] M. A. Persinger, B. T. Dotta, K. S. Saroka, World Journal of Neuroscience 3 (2013) 10-16. 
[5] M. A. Persinger, B. T. Dotta, K. S. Saroka, M. A. Scott, Journal of Consciousness Exploration \& Research 4 (2013) 1-14.

[6] B. T. Dotta, N. J. Murugan, L. M, Karbowski, M. A. Persinger, International Journal of Physical Sciences 8 (2013) 1783-1787.

[7] J. DeMeo, Water 3 (2011) 1-47.

[8] G. H. Pollack, Advances in Colloid and Interface Science 103 (2013) 173-196.

[9] B-h. Chai, J-m Zheng, Q. Zhao, G. H. Pollock, Journal of Physical Chemistry 112 (2008) 2242-2247.

[10] C. R. House, Water Transport in Cells and Tissues. Edward Arnold Ltd, London, 1974.

[11] N. J. Murugan, L. M. Karbowski, R. M. Lafrenie, M. A. Persinger, manuscript in submission, 2013.

[12] N. J. Murugan, L. K. Karbowski, R. M. Lafrenie, M. A. Persinger, PLOS ONE 8 (2013) e6174. doi: 10.371/journal.pone.0061714.

[13] M. A. Persinger, S. A. Koren, International Journal of Neurosciences 117 (2007) 157-175.

[14] E. Del-Giudice, Journal of Biological Physics 20 (1994) 105-116.

[15] M. A. Persinger, Journal of Physics, Astrophysics and Physical Cosmology 3 (2009) 1-3.

[16] L-C. Tu, J. Luo, G. T. Gillies, Reports on Progress in Physics 68 (2005) 77-130.

[17] M. A. Persinger, The Open Astronomy Journal 2 (2012) 125-128.

[18] H. Shibai, T. Maihara, Progress in Theoretical Physics 69 (1983) 77-88.

[19] A. Neronov, I. Vovk, Science 328 (2010) 73-75.

[20] J. Hofmann, M. Krug, N. Ortegel, L. Gerard, M. Weber, W. Rosenfeld, H. Weinfurter, Science 337 (2012) 72-75.

[21] B. Pakkenberg, H. J. G. Gundersen, Journal of Comparative Neurology 384 (1997) 312-320.

[22] M. A. Persinger, International Letters of Chemistry, Physics and Astronomy 8 (2013) 8-19.

[23] M A. Persinger, C. Lavallee, Journal of Consciousness Studies 19 (2012) 128-153.

[24] R. Llinas, U. Ribardy, Proceedings of the National Academy of Sciences 90 (1993) 2078-2081. 